\title{
The Volumetric Analysis of the Human Body as Starting Point for Clothing Pattern Design
}

\author{
Slavenka PETRAK, Ivana ŠPELIĆ, Maja MAHNIĆ NAGLIĆ
}

Faculty of Textile Technology, University of Zagreb, Croatia

DOI: $10.15221 / 18.083 \quad$ http://dx.doi.org/10.15221/18.083

\begin{abstract}
The paper presents evaluation of the overall and microclimatic volume changes due to different upper limb positions simulating functional reaching movements for the aircrew personnel. The study was performed in order to evaluate the needed ease allowance added to chest and waist circumference for outerwear garments in order to fully achieve the wearing comfort. The accurate 3D body scanning was used and the impact of the upper limb position on microclimatic volume distribution was tested. The scanning data process was performed using a 3D laser scanner and a computer analysis. The raw scans were processed and reconstructed. After the scan reconstruction, the volume and the area were calculated.

The experimental study covered the objective measuring methods: the material testing, the 3D scanning, the scan reverse engineering modelling and the volume/area calculation. The volume calculations included both the overall volume calculation for the unclothed torso and for the torso dressed in the chosen outerwear jacket. It also included the volume calculation of an air layer formed between second and third - layered garments.

The clothing ensemble CE 0 is the control variable, the unclothed body. The CE 1 is the clothing ensemble combined from the underlying basic garments (undershirt, underpants, classical male business shirt, and jeans) and combined with the bomber jacket. The three human subjects with the analogous body proportions (the height of $185 \mathrm{~cm}$ and chest girth of $100 \mathrm{~cm}$ ) were scanned using the 3D laser body scanner Vitus Smart (Human Solutions $\mathrm{GmbH}$ ) in an upright standing position according to ISO 20685:2010 changing only upper limb positions simulating functional reaching movements for the aircrew personnel.
\end{abstract}

Keywords: 3D body scanning, volumetric analysis, human body, clothing pattern design

\section{Introduction}

The adaptation in clothing represents one of the conscious mechanisms for body temperature control along with changing the activity. These factors are called the personal factors and they can provide the desired thermal comfort level [1]. One of the possible behavioral action is to alter the clothing in order to gain the thermal stability and to accomplish the thermal comfort within different environments. The function of clothing is to assure the thermal stability of the human body and the thermal insulation properties of the clothing play a crucial role for a human's heat maintenance [2,3]. Since the clothing thermal insulation is of the high significance in deriving the satisfactory thermal state of the human body, it should be analyzed with great care.

The clothing design is of the crucial factors in achieving the optimum thermal exchange rate between the human body and it's environment. The clothing has to enable the thermal exchanges between a man and his environment, maintained at a rate which will not cause the discomfort under vastly different environmental conditions. The two-dimensional fabrics are transformed into the three-dimensional clothing during development of the garments.

Since the heat transfer rate is determined by both the fabric selection as well as the microclimatic air layers formed under the clothing [4,5], which impact the volume of the garments and the ensembles, one has to determine an air layer which alters a heat transfer rate from the body to the environment $[6,7]$.

Among first authors to acknowledge the impact of the clothing in overall reduction of the conduction losses by trapping a still air within the fabric structures and between the garment layers, were the $\mathrm{E}$. A. McCullough and associates. They have proven the clothing to resists the convective heat losses by preventing convection currents forming next to the body and by providing a barrier against air currents in the environment [8]. The nonhomogeneous air layers of a different thickness are created under clothing. The type, the specific design and the fabric selection will determine the clothing fit to the body, thus substantially help to create the air layers under clothing. The variables affecting the distribution and volume of the air spaces within garments and ensembles are some physical characteristics of the fabrics, the configuration of the clothing in accordance to the posture and cover or wrapping arrangement, an activity of the wearer, and the ambient environmental conditions. 
The clothing design and style also affects the distribution and volume of the air spaces within garments and ensembles. One has to take into account the number of layers, the fit and the drape, the flexibility of the layers and the adequacy of closures, the surface area and the geometry [2].

The air, which serves as an insulator, gets trapped inside the clothing microclimate (as well as within the fabrics and between the layers of the fabrics). The clothing microclimate generally refers to the air layer nearest to the skin when people wear clothing [9]. The geometry of the surface changes and the stacking fabrics one over another may result in the additional air being enclosed between the layers of the fabrics during formation of the garments. Those air layers formed between the layers of the garments combined into the ensemble, which form the microclimate, are not uniformly distributed. The total insulation of clothing is defined as a thermal insulation from the body surface to an environment including all the clothing, the enclosed air layers and the boundary air layer [10]. According to that, the dry heat loss from the body is a combination of both the resistance provided by the clothing and from the entrapped microclimatic air, apropos the heat transferred by the exposed skin and the heat passing through the clothing [11].

The garment fit and resultant air volume is a crucial factor in determining thermal insulation value of clothing ensembles [5,12-14]. The thermal insulation will increase almost linearly with air gap amplification, as long no convection is present $[5,15,16]$. However, since the air layers trapped between garments have the complex geometry, quantification of the microclimatic air volume is a complex subject.

The air will become an ineffective insulator when exceeding a certain limit, it as the forced convection due to the body movements creating a bellows effect becomes pronounced thus preventing the linear increase of the total dry heat loss $[15,17,18]$. The properties of the underlying layers will affect the thermal resistance, including the number and the distribution of the air layers. A single large air space is less thermally resistant than a number of smaller air spaces of comparable total thickness due to the greater convective heat losses and the bellows in larger air spaces. Increasing the thickness of the inner layer fabrics also results in the thermal resistance change for as much $200 \%$ when the thickness of the inner layer fabrics is increased from 5 to $40 \mathrm{~mm}$ [2].

The thickness of the air and fabric layers can be quantified by subtracting nude body radius from the outer clothing radius, thus calculating the thickness of overall microclimatic layer [19]. The volume of the microclimatic layers can also be quantified by using 3D phase-shifting moiré topography, but no sufficient data on precise calculating volume of the air gaps was proven $[5,20]$.

The 3D scanning technique was proven to be most accurate and reproducible method for the air volume quantification under clothing [20] and is recently used to precisely quantify the volume of the microclimatic layers [21].

The purpose of the study is to conduct the volumetric analysis of the human body as starting point for clothing pattern design and to investigate the microclimatic volume increase under the third - layered clothing. In order to quantify volume of the microclimatic air formed under the jacket in the upper part of the body, the human subjects were scanned using the 3D laser body scanner since the 3D scanning method is the most accurate and reproducible method for the air volume quantification under clothing. The operating principle of the 3D laser scanning is projecting and measuring the reflection of a laser beam after striking an object that is presented to the laser in a way to engrid the whole surface. The 3D image of the object can be used to find the envelope volume as well as the surface area through postprocessing software. The present study will investigate the impact of the microclimatic air volume on the overall jacket volume (serves as the outerwear garment). Since none of the previous studies covered the volume enlargement due to the third layer of outerwear garment, in this study the volume under the jacket was calculated after the raw scan processing. Those results could be of a great help in the future to compare the impact of the microclimatic volume on the thermal insulation value of garments and ensembles by deducing the threshold volume, after which the thermal insulation will start to decrease.

\section{Methodology}

The first part of the experimental study covered the objective measuring methods: the material testing, the jacket construction and the 3D scanning. In the second part of the study the scans were reconstructed and processes with Geomagic Design X software and later imported to the Geomagic Verify software in order to calculate the volumes.

The volume calculations included both the overall volume calculation for the clothing ensemble CE 0 , which is the control variable and represents the unclothed body and the overall volume calculation for the CE 1 is the clothing ensemble combined from the underlying basic garments (undershirt, underpants, classical male business shirt, and jeans) and combined with the bomber jacket. The microclimatic air layer formed under the jacket has also been calculated. 
The three human subjects with the analogous body proportions (the height of $185 \mathrm{~cm}$ and chest girth of $100 \mathrm{~cm}$ ) were scanned using the 3D laser body scanner Vitus Smart (Human Solutions $\mathrm{GmbH}$ ) in an upright standing position according to ISO 20685:2010 [22].

According to ISO $3636: 1977$ and ISO $8559-2: 2017$ [23,24], it is classified as a slim figure. These standards define the male slim figure in terms of a difference of at least $14 \mathrm{~cm}$ between chest and waist circumference and $16 \mathrm{~cm}$ difference between waist and hip circumference. They were selected to meet the height requirement for tall figure assigned by BS EN $13402-3$ [25] and the body measurements compliant with the athletic posture according to ISO/TR 10652:1991 [26]. The subjects' dimensions ranged as - body height $183-184 \mathrm{~cm}$, chest girth $98-102 \mathrm{~cm}$; waist girth $80-83 \mathrm{~cm}$; hip girth $99-102$ $\mathrm{cm}$. The body mass ranged $78-79$, which sets BMI interval ranging $23.0-23.3$. The three human subjects were scanned changing only upper limb positions simulating functional reaching movements for the aircrew personnel. Six types of the functional arm reaching positions were chosen, Figure 1:

1) reference starting arm position (with arms hanging by the side of the body with the elbows straight and with the palms of the hand facing inward),

2) vertical superior functional reach when arms are vertically flexed and abducted from the shoulder joint and raised above the head in the sagittal plane about the frontal axis (simulating full upper extension of the arm in vertical position in order to change upper controls placed above the head or respectively the maximum height of controls inside the aircraft cabin above the seat surface),

3) overall lateral limit of reach when arms are horizontally abducted and extended to the side of the body from the shoulder joint in the frontal plane about the sagittal axis (if controls are to be operated sideways with fingertips),

4) forward anterior functional reach when arms are horizontally flexed from the shoulder joint in front of the body in the sagittal plane about the frontal axis (which represents the distance from the back of the shoulder to the thumb - tip of a pinch grip or respectively the maximum forward location of controls for easy operation),

5) elbow functional reach when arms are flexed in the elbow joint in the sagittal plane about the frontal axis (maximum forward location of the controls for operation with the upper arm vertical and forearm horizontal) and

6) backward posterior functional reach, where arms are horizontally hyperextended to the back of the body from the shoulder joint in the sagittal plane about the frontal axis (there are no controls positioned at the back of the pilot seat in the cabin of the aircraft or the helicopter, but with this arm movement, the jacket will stretch over the chest and the air will compress).

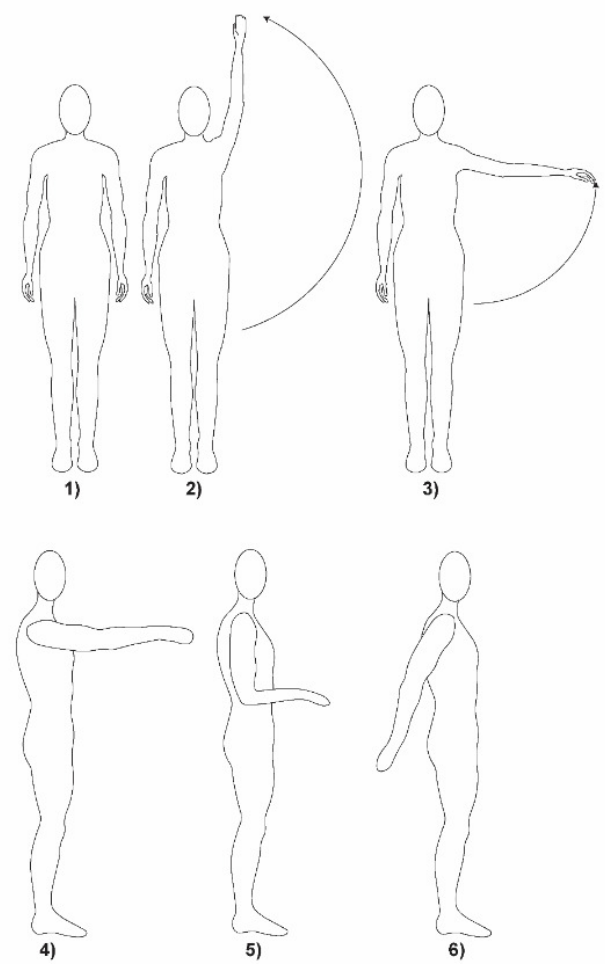

Fig. 1. Upper body scanning and arm functional arm reaching positions: 1) reference starting arm position, 2) vertical superior functional reach, 3) overall lateral limit of reach, 4) forward anterior functional reach, 5) elbow functional reach and 6) backward posterior functional reach 
Each subject was scanned trice, firstly dressed in a tight underwear and afterwards dressed in the selected ensemble with jacket added as the outerwear garment. The raw scans were processed and reconstructed by two different $3 D$ CAD tools for design, engineering and preparation for the manufacturing as well for the scan modelling, Figure 2. The first software packages used to process scans are Geomagic Design X and Geomagic Verify software by 3D Systems Inc.

\subsection{The jacket preparation}

Both the microclimatic and the overall volume under garments was calculated. The model of the jacket, later forming the clothing ensemble CE 1, was constructed and produced and the amount of the ease allowance was added strictly to the chest $(100 \mathrm{~cm})$ and the waist $(82 \mathrm{~cm})$ circumference.

The final chest circumference of the jacket was $122 \mathrm{~cm}(22 \mathrm{~cm}$ of the ease allowance), waist circumference $118 \mathrm{~cm}$ (36 cm of the ease allowance) and hip circumference was $104 \mathrm{~cm}$ in order to get a tight fitting jacket in the hip area. The shoulder seam, the neck opening, the hip circumference, the length of the sleeves and the length of the jacket maintained uniform.

The thermal resistance of garments is related to the garment volume since the amount of the body surface covered by garments is greater with the ease allowance augmentation. Since the jackets as outerwear garments usually have greater ease allowances, they provide greater microclimatic volume amplification.

The specific model is bound at the bottom, the rate and the volume of the air exchanged between the assembly microclimate and the ambient environment will not be affected by the extent of the air flow through the openings in the garment. The CV 0 is the control variable with subjects being scanned in tight underwear with different arm positions, Figure 2.

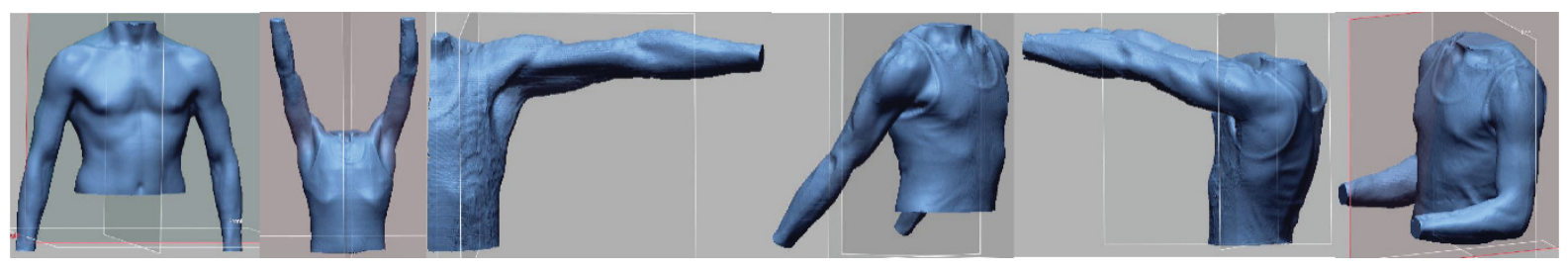

Fig. 2. The reconstructed body of the scanned human body,

also known as the control variable CV 0 with different arm positions

Since the fabrics provide resistance to heat flow, the thermal properties of the fabrics were tested. The conductive and radiative heat fluxes between the fabric layers, act in parallel across the trapped air layer. The outer shell for jacket was made of the three - layered laminate (outer layer and inner lining $100 \%$ polyester - PES, the middle layer polytetrafluoretilen membrane - PTFE protected with polyurethane particles - PU). The lining was 100\% PES fabric. The materials testing was performed according to ISO 3801:1977 (the mass per unit area) [27], ISO 5084:1996 (the thickness - h) [28], ISO 9237:1995 (an air permeability - AP) [29] and ISO 11092:2014 (the water vapour resistance - Ret, and the thermal resistance $-\mathrm{R}_{\mathrm{ct}}$ at $\mathrm{RH}=60 \%$ ) [30]. The structural and physical characteristics of materials selected for jacket production and garments are seen in Table 1.

Table 1. Characteristics of materials selected for jacket production

\begin{tabular}{|l|c|c|c|c|c|}
\hline $\begin{array}{l}\text { Selected } \\
\text { materials }\end{array}$ & $\begin{array}{c}\text { Mass per unit } \\
\text { area }\left[\mathrm{kg} / \mathrm{m}^{2}\right]\end{array}$ & $\mathrm{h}[\mathrm{mm}]$ & $\mathrm{AP}\left[\mathrm{kg} / \mathrm{m}^{2}\right]$ & $R_{\mathrm{et}}\left[\mathrm{m}^{2} \mathrm{~Pa} / \mathrm{W}\right]$ & $\mathrm{R}_{\mathrm{ct}}\left[\mathrm{m}^{2} \mathrm{~K} / \mathrm{W}\right]$ \\
\hline outer fabric & $1.89^{*} 10^{-5}$ & 0.3800 & 0 & 9.3767 & 0.0706 \\
\hline lining & $0.55^{\star} 10^{-5}$ & 0.0776 & 121.4 & 10.0148 & 0.0677 \\
\hline
\end{tabular}

\subsection{The scan Post-processing and volume calculation}

3D scanners generate polygonal approximation to human model [31]. To quantify the volume of the microclimatic layers under jacket, the chosen human subjects were scanned using the 3D laser body scanner Vitus Smart (Human Solutions $\mathrm{GmbH}$ ) in an upright standing position. Vitus Smart is a noncontact active and whole-body 3D scanner. Non-contact active 3D scanners use a laser to illuminate the object surface to measure the distances or to recognize surficial curves. The whole-body 3D scanners are equipped with four wide-view, high-resolution scanners, which rotate around the person 
to scan every angle. This high-powered precision scan is able to capture even the smallest details, such as hair, wrinkles on clothes, and buttons. The scanning process generates millions of triangulated surfaces. Even though 3D scanners provide accurate and detailed geometric data from real-world objects, they are restricted to producing a discrete representation due to irregularity, discontinuity, huge dataset size, and missing areas [31].

Bearing that in mind, each dressed subject was scanned trice firstly dressed in a tight underwear and afterwards dressed in one of the selected clothing ensemble. Since the scanning technology enables the polygonal mesh generation (a network of connected triangular elements to wrap the object surface) thus reducing the point cloud data without compromising the surface quality, the rendering and determination of volume and surface area can be obtained.

The raw scans were saved in obj. format. The multiple scanned OBJ - formats imported by the Geomagic Design X software, to be processed and reconstructed, Figure 3. The Geomagic Design X software is 3D CAD tool for design, engineering and preparation for the manufacturing as well for the scan modelling. Three separate scans of each clothed subject dressed in the same selected jacket were imported and aligned by assigning corresponding alignment points between two scans. Each scan consists of the eight scanned meshes. After the scans have been aligned, they were combined into a single fused mesh and the scan reconstruction was performed.

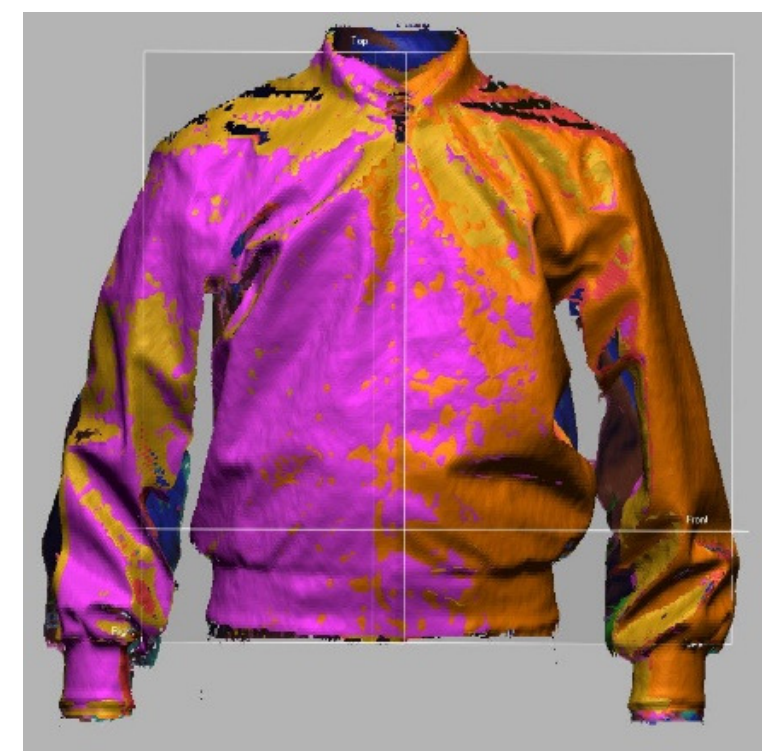

Fig. 3. The raw scan imported as obj. format

The first important stage of the scan processing is the reverse modelling procedure. Since the scanned human body covered in clothing has the complicated geometry, many of the surface and the mesh features are imperfect and should be reconstructed using the remaining polygons, Figure 4.

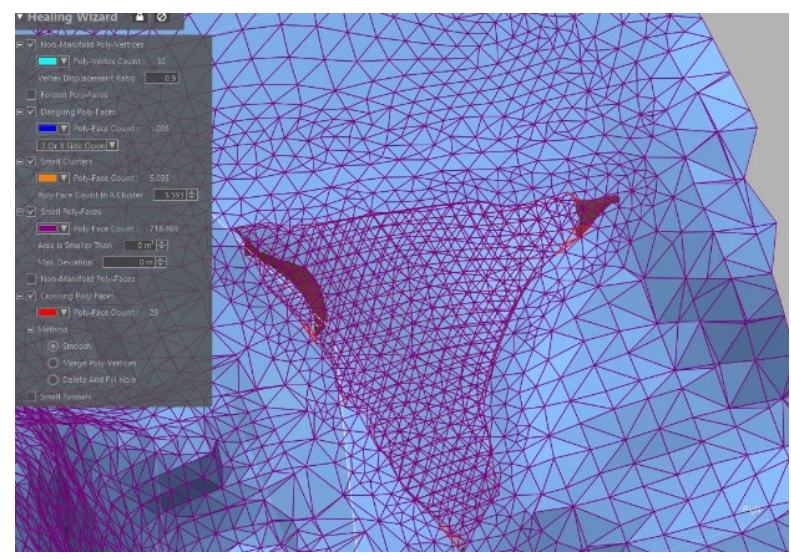

Fig. 4. The scan reconstruction 
After the scans have been aligned, one should combine them into a single fused mesh, Figure 5. Although the Geomagic Design X software has the ability to automatically clean - up the point cloud data, analyze the mesh and repair the scan, it is impossible to automatically re - mesh the complex geometry of the dressed human body.

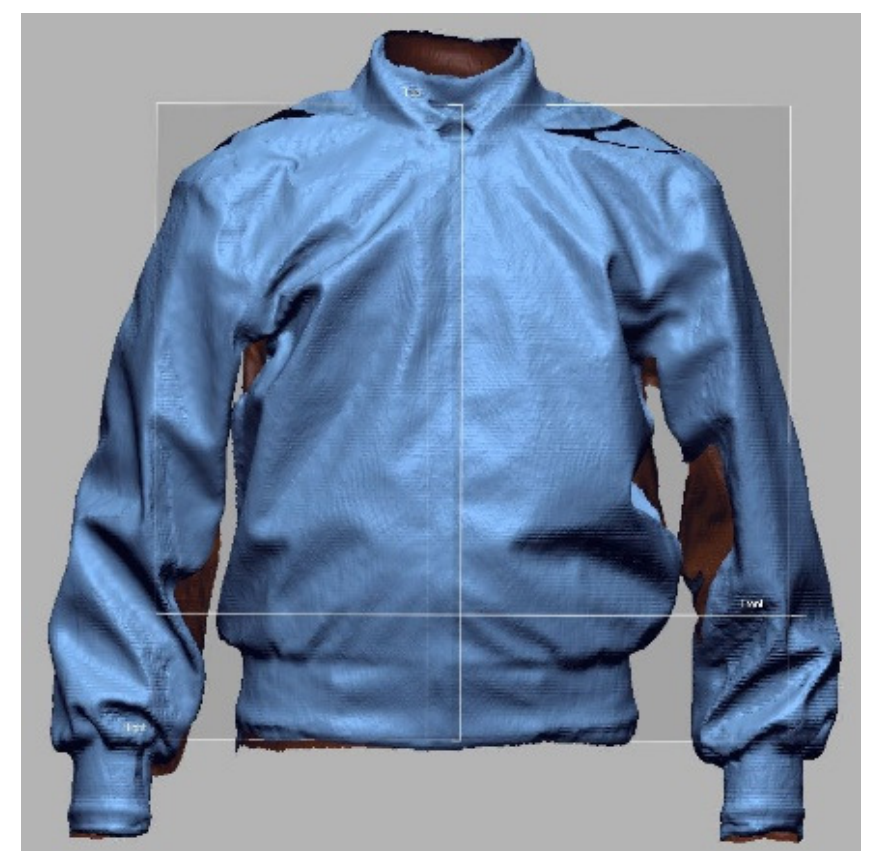

Fig. 5. The scan after the mesh merge

The reverse modelling and the building was done manually in stages. The imported obj. files were processed and edited manually through stages: 1) scan alignment and merging into the single model, 2) mesh preparation by filling holes and healing defects, 3) missing area restoration, 4) re - meshing and rewrapping and 5) surface smoothing. After conducting extraction of the jacket' scans in different arm positions, the scanned data processing and the manual editing phase, the scans were automatically finished by choosing the mesh build-up wizard. Once the model is finished, it can be exported or saved for further usage, Figure 6.

The total volume of the scanned jacket was calculated by subtracting the volume of the outer clothing layer and the volume of the undressed torso. The volume and an area measurements of the scans were determined by a Geomagic Verify software. The NIST expanded uncertainty (with coverage factor $\mathrm{k}=2$ ) for Geomagic softwares is less than 1x10-9. The deviation results are less than $1 \mu \mathrm{m}$. with level of confidence of $95 \%$ [32].

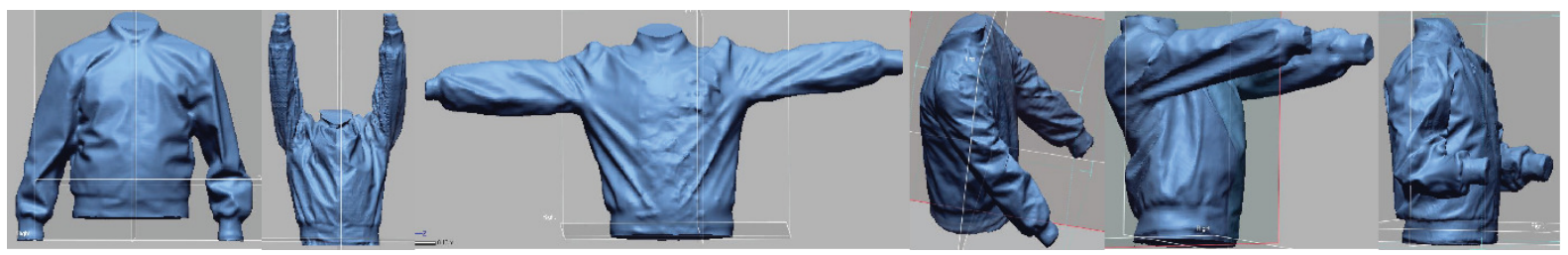

Fig. 6. Processed scans of male subject dressed in selected clothing ensemble with different arm positions

\section{Results and discussion}

The mean volumes of the scanned objects were calculated for each jacket. Each subject was scanned three times dressed in one jacket always in the same posture. One scanned jacket was reconstructed by merging three repeated consecutive scans, bearing in mind that each scan has altogether eight meshes. The area (ACG) and the volume (VCG) of unclothed torso and torso dresses with the jacket as the outerwear garment combined with underlying garments (CE1), was calculated as the mean value for all three subjects as seen in Table 2 . 
Table 2. The calculation of the mean value of area (ACG) and the volume (VCG) for different scanned arm positions together with variances using the Reverse Engineering software Geomagic Verify Viewer

\begin{tabular}{|l|c|c|c|c|}
\hline Scanned object & AcG $\left[\mathrm{m}^{2}\right]$ & $\sigma$ & $\mathrm{V}_{\mathrm{CG}}\left[\mathrm{dm}^{3}\right]$ & $\sigma$ \\
\hline Unclothed torso, position 1, hands beside & 0,8029 & $2,33 \times 10^{-8}$ & 34,49 & 0,00123 \\
\hline Unclothed torso, position 2, hands above & 0,8478 & $1,19 \times 10^{-7}$ & 35,90 & $3,33 \times 10^{-5}$ \\
\hline Unclothed torso, position 3, hands apart & 0,9295 & $1,59 \times 10^{-7}$ & 28,08 & 0,00023 \\
\hline Unclothed torso, position 4, hands front & 0,8192 & $4,00 \times 10^{-8}$ & 33,41 & 0,00063 \\
\hline Unclothed torso, position 5, front bend & 0,7871 & $3,99 \times 10^{-8}$ & 34,11 & 0,00063 \\
\hline Unclothed torso, position 6, hands back & 0,7898 & $2,33 \times 10^{-8}$ & 33,75 & 0,00063 \\
\hline Torso dressed in CE 1, position 1, hands beside & 1,2487 & $2,33 \times 10^{-8}$ & 66,81 & 0,00123 \\
\hline Torso dressed in CE 1, position 2, hands above & 1,1724 & $2,33 \times 10^{-8}$ & 52,47 & 0,00043 \\
\hline Torso dressed in CE 1, position 3, hands apart & 1,2451 & $4,00 \times 10^{-8}$ & 64,18 & 0,00163 \\
\hline Torso dressed in CE 1, position 4, hands front & 1,1209 & $3,33 \times 10^{-9}$ & 56,47 & 0,00093 \\
\hline Torso dressed in CE 1, position 5, front bend & 1,2734 & $2,33 \times 10^{-8}$ & 62,38 & 0,00143 \\
\hline Torso dressed in CE 1, position 6, hands back & 1,1872 & $6,33 \times 10^{-8}$ & 64,21 & 0,00120 \\
\hline
\end{tabular}

The lowest value of the area was calculated for the unclothed torso in referent position 5 , representing the elbow functional reach when arms are flexed in the elbow joint in the sagittal plane about the frontal axis. The highest value of the area was calculated for the unclothed torso was calculated for the position 3 , representing the overall lateral limit of reach when arms are horizontally abducted and extended to the side of the body from the shoulder joint in the frontal plane about the sagital axis. As for the dressed body the lowest volume was calculated for position 3 and the highest value was calculated for the position 2, which is the vertical superior functional reach when arms are vertically flexed and abducted from the shoulder joint and raised above the head in the sagittal plane about the frontal axis.

The lowest value of the area was calculated for the unclothed torso was calculated for the position 4, representing the forward anterior functional reach when arms are horizontally flexed from the shoulder joint in front of the body in the sagittal plane about the frontal axis. The highest value of the area of the unclothed body was calculated for the position 5, representing the elbow functional reach when arms are flexed in the elbow joint in the sagittal plane about the frontal axis.

The lowest value of the volume for the dressed torso was calculated for the position 2, representing the vertical superior functional reach when arms are vertically flexed and abducted from the shoulder joint and raised above the head in the sagittal plane about the frontal axis. The highest volume value for the dressed torso was calculated for the position 1, with reference starting arm position.

The added jacket as the outerwear garment caused simultaneous air volume amplification in the microclimatic area. The increase in the volume of the microclimatic air layer (VMAL) underneath the jacket was calculated in comparison to nude torso as the mean value for all three subjects as seen in Table 3.

Table 3. The calculation of the mean value of the volume of the microclimatic air layer (VMAL) and the percentage of the area/volume increase in comparison to unclothed torso

\begin{tabular}{|c|c|c|c|}
\hline Position & $\begin{array}{c}\text { The volume of the } \\
\text { microclimatic air } \\
\text { layer [dm }{ }^{3} \text { ] }\end{array}$ & $\begin{array}{c}\text { The percentage of the area } \\
\text { increase between in comparison } \\
\text { to unclothed torso [\%] }\end{array}$ & $\begin{array}{c}\text { The percentage of the volume } \\
\text { increase between in comparison to } \\
\text { unclothed torso [\%] }\end{array}$ \\
\hline 1 & 32,32 & 55,52 & 93,71 \\
\hline 2 & 16,57 & 38,29 & 46,16 \\
\hline 3 & 36,10 & 33,95 & 128,56 \\
\hline 4 & 30,46 & 50,32 & 90,25 \\
\hline 5 & 28,27 & 61,78 & 82,88 \\
\hline 6 & 23,06 & 36,83 & 69,02 \\
\hline
\end{tabular}


The percentage of the microclimatic area and volume increase for each arm position was calculated in comparison to the unclothed torso. The interesting part is how much does the position of the hands affects the volume and the area calculation. As seen from the calculation the highest amplification in the percentage of the volume increase in comparison to unclothed torso was seen for position 3 . This is the overall lateral limit of reach when arms are horizontally abducted and extended to the side of the body from the shoulder joint in the frontal plane about the sagittal axis. The increase in the volume of the microclimatic air layer for $36,10 \mathrm{dm}^{3}$ is equal to $128,56 \%$ of the volume increase between in comparison to unclothed torso. The lowest value of the volume of the microclimatic air layer $\left(16,57 \mathrm{dm}^{3}\right)$ was calculated for reference starting arm position 2, which represents the vertical superior functional reach when arms are vertically flexed and abducted from the shoulder joint and raised above the head in the sagittal plane about the frontal axis.

The highest percentage of the area increase between in comparison to unclothed torso was calculated for position 5 where arms are horizontally hyperextended to the back of the body from the shoulder joint in the sagittal plane about the frontal axis $(61,78 \%)$. The lowest percentage of the area increase between in comparison to unclothed torso was calculated for position 3 simulating the overall lateral limit of reach when arms are horizontally abducted and extended to the side of the body from the shoulder joint in the frontal plane about the sagittal axis (33,95\%).

\section{Conclusions}

The evaluation of the jacket volume changes due to different upper limb positions simulating functional reaching movements for the aircrew personnel was analysed. The study points to great need for evaluating the appropriate position and posture when scanning human subjects. Different functional arm reaching positions were chosen for standing subjects scanned by 3D scanner. After processing and reconstructing the raw scans by two different $3 D$ CAD tools for the scan reverse modelling, the mean values of area (ACG) and the volume (VCG) for different scanned arm positions were calculated. The calculation of the mean value of the volume of the microclimatic air layer (VMAL) and the percentage of the area/volume increase in comparison to unclothed torso was also performed. The results point to need for choosing the appropriate scanning position in accordance with the final calculated variable. If the calculation of the clothing area is in order, the best scanning position observed was the position 6 where arms are horizontally hyperextended to the back of the body from the shoulder joint in the sagittal plane about the frontal axis. If the calculation of the clothing volume is in order, the best scanning position observed was the position 2 , when arms are vertically flexed and abducted from the shoulder joint and raised above the head in the sagittal plane about the frontal axis.

The complex geometry of dressed human body makes the quantification of the microclimatic air volume, the overall area and the volume of clothing an interesting field of research as the basis for further clothing pattern design. Especially when considering the volume of air trapped inside the clothing serving as the thermal insulator or when considering the surface area factor or the convective heat transfer area of clothing, since the radiative and convective heat transfer coefficient depend on body postures. In the future, the reverse engineering and scan modelling shall also be performed by other 3D CAD software in order to compare the data, modelling procedures and to gain insight on the most precise method to prepare the complex human body models set upon 3D scanning procedure.

\section{References}

[1] M. Prek. Thermodynamical analysis of human thermal comfort. Energy, 31, (2006), p. 732-743, doi:10.1016/j.energy.2005.05.001

[2] C. A. Wilson, R. M Laing, D. J. Carr. Air and Air Spaces - the Invisible Addition to Thermal Resistance. Journal of the Human-Environmental System, Vol. 5, No. 2, 2002, pp. $69-77$

[3] F. R.d'Ambrosio Alfano, B. W. Olesen and B. I. Palella. Povl Ole Fanger's Impact Ten Years Later, Energy and Buildings, (2017), http://dx.doi.org/10.1016/j.enbuild.2017.07.052

[4] Advances in the dyeing and finishing of technical textiles. Woodhead Publishing Series in Textiles: No. 138, Gulrajani, M. L. (Ed.), Woodhead Publising Ltd., ISBN 978085709-339, Cambridge, United Kingdom, (2013)

[5] Lee, Y., Hong, K. \& Hong, S. - A.: 3D quantification of microclimate volume in layered clothing for the prediction of clothing insulation, Applied Ergonomics, 38 (2007), pp. 349-355

[6] ASHRAE Handbook Fundamentals (SI ed.). ch. 8. (2005). Atlanta, United States of America: American Society of Heating, Refrigerating, and Air Conditioning Engineers. ISBN-10: 1883413885

[7] Havenith, G., Richards, M. G. M., Wang, X., Bröde, P., Candas, V., den Hartog E, Holmér, I., Kuklane, K., Meinander, H \& Nocker, W. (2008). Apparent latent heat of evaporation from clothing: attenuation and "heat pipe" effects, Journal of Applied Physiology, 104 (1), 142 - 149 
[8] McCullough E A and Jones B W. A comprehensive data base for estimating clothing insulation, IER technical report no. 84-01, ASHRAE, Inc, 1984

[9] Kwon, J. \& Choi, J.: Clothing insulation and temperature, layer and mass of clothing under comfortable environmental conditions, Journal of Physiological Anthropology, 32 (2013) 1, doi: 10.1186/1880-6805-32-11

[10] ISO 9920:2009 Ergonomics of the thermal environment - Estimation of thermal insulation and water vapour resistance of a clothing ensemble, ISO-International Organization for Standardization, 2009

[11] Tuğrul Oğulata ,R. (2007). The Effect of Thermal Insulation of Clothing on Human Thermal Comfort. Fibres \& Textiles in Eastern Europe, $15(2,61)$

[12] Zhang, Z. \& Li, J. (2011). Volume of Air Gaps under Clothing and Its Related Thermal Effects. Journal of Fiber Bioengineering \& Informatics, 4 (2), 137-144

[13] Zhang, Z. - H., Wang, Y. \& Li, J. (2011). Model for Predicting the Effect of an Air Gap on the Heat Transfer of a Clothed Human Body. Fibres \& Textiles in Eastern Europe, 19, (4-87), 105-110

[14] McQuerry, M., DenHartog, E. \& Barker, R. (2017). Analysis of air gap volume in structural firefighter turnout suit constructions in relation to heat loss, Textile Research Journal, 0(00), 1-10, DOI: $10.1177 / 0040517517723024$

[15] Mert, E., Psikuta, A., Bueno M. - A., Rossi, R. M. (2015a). Effect of heterogenous and homogenous air gaps on dry heat loss through the garment. International Journal of Biometeorology, 59, 17011710, DOI: 10.1007/s00484-015-0978-x

[16] Mert, E., Böhnisch, S., Psikuta, A., Bueno M. - A. (2015b). Determination of the Air Gap Thickness underneath the Garment for Lower Body Using 3D Body Scanning. In: Proceedings of the 6th International Conference on 3D Body Scanning Technologies. D'Apuzzo, N. (Ed.). Homometrica Consulting (Lugano, Switzerland), 114-119

[17] Clark, R. P., de Calcina-Goff, M. L. (2009). Some aspects of the airborne transmission of infection. Journal of the Royal Society Interface, 6(6), 767-782

[18] Havenith, G. (2005). Clothing heat exchange models for research and application. In: Proceedings of 11th International Conference on Environmental Ergonomics. Holmer, I., Kuklane, K., Gao, C. (Ed.). Lund University (Ystad, Sweden), 66-73

[19] McCullough, E.A. \& Hong, S. (1994) A data base for determining the decrease in clothing insulation due to body motion. ASHRAE Transactions, 100, 765-775

[20] Daanen, H., Hatcher, K., Havenith, G. (2002). Determination of clothing microclimate volume. In: Proceedings of the 10th International Conference on Environmental Ergonomics. Tochihara, Y., Ohnaka. T. (Ed.). Kyushy Institute of Design (Fukuoka, Japan), 665-668

[21] Špelić I., Rogale D., Mihelić-Bogdanić, A.: The laboratory investigation of the clothing microclimatic layers in accordance with the volume quantification and qualification, The Journal of The Textile Institute, 2018, DOI: 10.1080/00405000.2018.1462087

[22] ISO 20685:2010 3-D scanning methodologies for internationally compatible anthropometric databases, ISO-International Organization for Standardization, 2010

[23] ISO 3636:1977 Size designation of clothes - Men's and boys' outerwear garments, ISOInternational Organization for Standardization, 1977

[24] ISO 8559-2:2017 Size designation of clothes - Part 2: Primary and secondary dimension indicators, ISO-International Organization for Standardization, 2017

[25] BS EN 13402 - 3:2017 Size designation of clothes. Size labelling based on body measurements and intervals, ISO-International Organization for Standardization, 2017

[26] ISO/TR 10652:1991 Standard sizing systems for clothes, ISO-International Organization for Standardization, 1991

[27] ISO 3801:1977 Textiles - Woven fabrics - Determination of mass per unit length and mass per unit area, ISO-International Organization for Standardization, 1977

[28] ISO 5084:1996 Textiles - Determination of thickness of textiles and textile products, ISOInternational Organization for Standardization, 1996

[29] ISO 9237:1995 Textiles - Determination of the permeability of fabrics to air, ISO-International Organization for Standardization, 1995

[30] ISO 11092:2014 Textiles -- Physiological effects - Measurement of thermal and water-vapour resistance under steady-state conditions, ISO-International Organization for Standardization, 2014

[31] S.-H., Yoon and J. Lee: Computing the Surface Area of Three-Dimensional Scanned Human Data, Symmetry 2016, 8, 67; doi:10.3390/sym8070067

[32] Report of special test, NIST Test No: 681/280055-10 for Geomagic Inc. (2010). Maryland, USA: United States Department of Commerce, National Institute of Standards and Technology 